\title{
Vestibular Signals of Posterior Parietal Cortex Neurons during Active and Passive Head Movements in Macaque Monkeys
}

\author{
FRANÇOIS KLAM AND WERNER GRAF \\ Laboratory de Physiologie de la Perception et de l'Action, CNRS/Collège de France, \\ 11, place Marcelin Berthelot, 75231 Paris Cedex 05, France
}

\begin{abstract}
The posterior parietal cortex may function as an interface between sensory and motor cortices and thus could be involved in the formation of motor plans as well as abstract representations of space. We have recorded from neurons in the intraparietal sulcus, namely, the ventral and medial intraparietal areas (VIP and MIP, respectively), and analyzed their head-movementrelated signals in relation to passive and active movements. To generate active head movements, we made the animals track a moving fixation spot in the horizontal plane under head-free conditions. When under certain circumstances the animals were tracking the fixation spot almost exclusively via head movements, a clear correlation between neuronal firing rate and head movement could be established. Furthermore, a newly employed paradigm, the "replay method," made available direct comparison of neuronal firing behavior under active and passive movement conditions. In such case, the animals were allowed to make spontaneous head movements in darkness. Subsequently, the heads were fixed and the previously recorded active head-movement profile was reproduced by a turntable as passive stimulation. Neuronal responses ranged from total extinction of the vestibular signal during active movement to presence of activity only during active movement. Furthermore, in approximately one-third of the neurons, a change of vestibular on-direction depending on active versus passive movement mode was observed, that is, type I neurons became type II neurons, etc. We suggest that the role of parietal vestibular neurons has to be sought in sensory space representation rather than reflex behavior and motor control contexts.
\end{abstract}

KEYWORDS: parietal cortex; vestibular; monkey; head movement; active movement; efference copy; self-motion perception

\section{INTRODUCTION}

Distinction between active and passive movements is an important function in everyday life for goal directed movements without interference by reflex mechanisms, and to allow vital reflexes to happen when necessary. In this context, seminal con-

Address for correspondence: Werner Graf, CNRS-LPPA, Collège de France, 11, place Marcelin Berthelot, 75231 Paris Cedex 05, France. Voice: +33-1-44-27-16-30; fax: 33-1-44-27-13-82. werner.graf@college-de-france.fr

Ann. N.Y. Acad. Sci. 1004: 1-12 (2003). (C) 2003 New York Academy of Sciences.

doi: 10.1196/annals.1303.001 
cepts have been developed, such as the reafference principle ${ }^{1}$ about how we move about and control and correct our own movements.

Recent studies on vestibular nuclei neurons during passive and active head movements showed that vestibular signals were strongly influenced by self-generated movements as early as the first vestibular projection neurons, ${ }^{2-4}$ and furthermore neurons in the vestibular nuclei related to head and eye movements subserving the vestibuloocular reflex are found in a similar proportion as neurons that signal only head velocity, without any eye-movement relatedness. ${ }^{3}$ The latter are thought to be part of the vestibulocortical relay. However, some vestibuloocular neurons project to thalamic units that, in turn, then project to the vestibular cortices. ${ }^{5}$ Vestibular thalamic and cortical units have been reported not to carry eye-movement signals. ${ }^{6-8}$

Several "vestibular" areas have been identified in the parietotemporal cortex of macaque monkeys, namely, the parietoinsular vestibular cortex, ${ }^{9}$ area $2 \mathrm{v}$ at the anterior tip of the intraparietal sulcus, ${ }^{10,11}$ area $3 \mathrm{a}^{12}$ as part of somatosensory area 3 , and in the posterolateral part of area PG. ${ }^{13}$ Recent anatomical studies confirmed and extended previous findings that the ventral intraparietal area (VIP) in the fundus of the intraparietal sulcus ${ }^{14}$ receives direct projections from vestibular areas and thus is part of a cortical vestibular network. ${ }^{15-17}$ This work extends our previous reports on vestibular responses in VIP. ${ }^{18-20}$ The principal aim of this study was to analyze head-movement-related signals in intraparietal vestibular neurons in relation to passive and active movements, in particular, because there exist large differences between active and passive movements as early as second-order vestibular neurons, ${ }^{2-4}$ and also with respect of the involvement of the parietal cortex in self-motion perception and representation of extrapersonal space.

\section{METHODS}

Extracellular recordings were made in the left hemispheres of two macaque monkeys, one male rhesus (Macaca mulatta) and one female fascicularis monkey (Macaca fascicularis). Animal care (housing, nourishment, veterinary consultations, surgical procedures, postoperative care, daily care) conformed to French government regulations (Ministries of Agriculture and Research, CNRS: approval 75-546) and European Union standards (European Communities Council Directive 86/609/EEC).

\section{Animal Training}

Head-fixed animals initially were trained to fixate a small spot of light within a narrow target window $\left(2 \times 2^{\circ}\right)$ for a certain time. The light spot could be kept stationary in darkness and in light to monitor a given neuron's resting activity. To determine a neuron's eye position sensitivity, we moved the spot in random order into nine different locations on the tangent screen. ${ }^{21}$ The spot could also be moved to test smooth pursuit sensitivity (FIG. 1A). The animals' heads were fastened in a specialized head-holder system that allowed free head movements about the vertical axis (horizontal head movements) (FIG. 1B). Once the head was freed, the animals were allowed either to make spontaneous head movements or to track the light spot via head or combined eye-head movements. From the combined signals, head, eye, and gaze information could be derived. 
In addition, we used a new testing paradigm to compare vestibular signals during active and passive head movements, the "replay method" (see also Robinson \& Tomko ${ }^{22}$ ). To that end, the animal was first allowed to make spontaneous head movements in the dark. Neuronal activity and the head-movement profile were recorded for various periods (up to $250 \mathrm{~s}$ ). After that, the animal's head was fixed, and
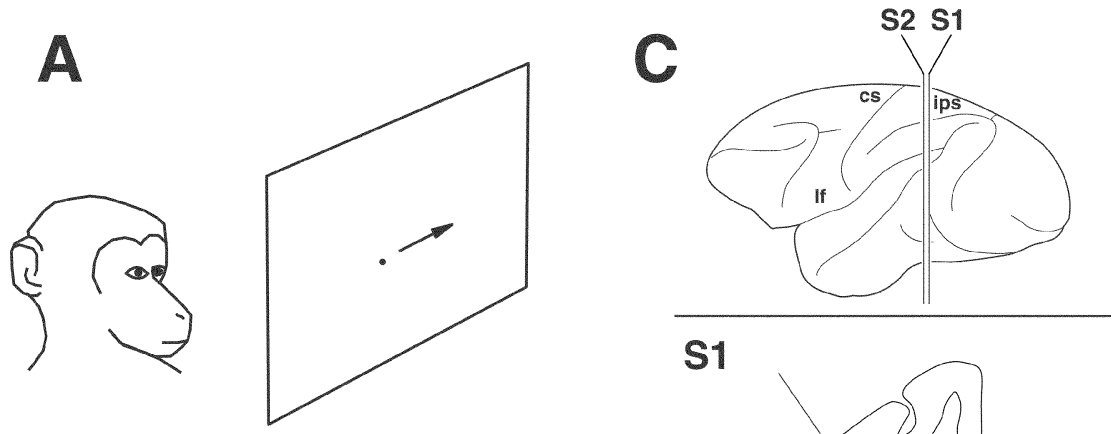

\section{Smooth pursuit}
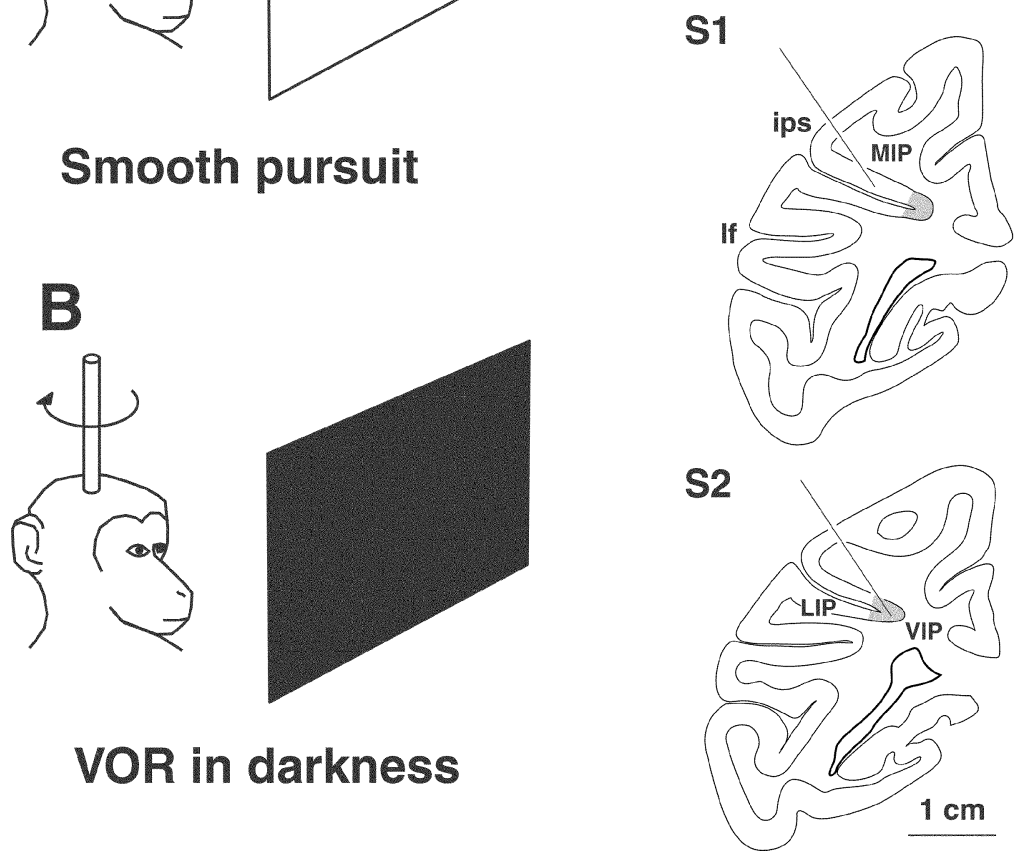

FIGURE 1. Stimulation parameters and location and reconstruction of recording sites. (A) Smooth pursuit: the animal was seated stationary and head-fixed in front of a tangent screen. It had to follow a light spot that was moved in eight cardinal linear directions. (B) Vestibular stimulation: the animal was rotated about the vertical axis in the darkened laboratory eliciting vestibular nystagmus. (C) Overall lateral view of the left hemisphere of a macaque monkey indicating the topographical relationship of cortical landmarks, that is, intraparietal sulcus (ips), central sulcus (cs), and the lateral fissure (lf). Vertical lines indicate the placement of two coronal sections (S1 and S2) shown below with reconstructed typical electrode tracks and recording sites (MIP, VIP) in the intraparietal sulcus. Area VIP is highlighted on the two slices in gray. Note that to reach VIP, electrode penetrations in some cases had to cross the intraparietal sulcus (LIP, lateral intraparietal area). 
the previously recorded active head-movement trajectory was reproduced by the turntable, again recording neuronal activity. Thus, a direct comparison during active and passive neuron discharge became available.

\section{Recordings}

Single cells were recorded extracellularly with glass-coated tungsten microelectrodes (F. Haer) in areas VIP and medial intraparietal area (MIP) of the two left hemispheres of the two monkeys. The animals were awake and performed several oculomotor tasks. Neurons were classified as located in area VIP on the basis of the recording sites and depth within the intraparietal sulcus, and for their response properties. ${ }^{14,23-26}$ Neurons in MIP were characterized by absent or low visual sensitivity and strong somatosensory responses located on the fingers, hands, and forearms. In a typical recording session, the passage of the electrode from MIP into VIP was marked by a distinct change in background and resting activity of the recorded neuronal elements.

Eye movements were recorded with the magnetic search-coil method, head movements with a head-holder mounted potentiometer. Previous tests had shown that the eye-movement signal remained linear within the range of the monkey's head movements. Neuronal signals were sampled at $1000 \mathrm{~Hz}$, and eye and head position at $250 \mathrm{~Hz}$.

\section{STIMULATION AND CHARACTERIZATION OF NEURONAL RESPONSIVENESS}

Vestibular stimulation was delivered via a vertical axis turntable (horizontal rotation) that could be moved manually or via a servo controller. To exclude any visual influence on vestibular responses during purely vestibular testing, we covered the animals' eyes with opaque pads and darkened the laboratory. During vestibular testing, the animals, naturally, had to be left free to make compensatory eye movements (vestibuloocular reflex [VOR]) (FIG. 1B). Fixation and smooth pursuit targets were back-projected onto a translucent tangent screen. Directional selectivity was assessed as described previously. ${ }^{27}$

\section{Data Analysis}

Vestibular responses were evaluated according to their preferred, or ondirections $^{28}$ (type I, II, and III), for the response strength and the response latencies under the two movement conditions. Vestibular on-directions are referred according to the recording sites in the left hemisphere, that is, a neuron that reacts with excitation during leftward (ipsilateral) rotation is defined as a type I neuron, etc.

Because reflex compensatory eye movements had to be allowed in our experimental tasks (VOR), eye-movement sensitivities were evaluated separately. When judging their effect on the vestibular response by adding them in a simple multilinear regression, the $\mathrm{R}^{2}$ never gained more than $10 \%$ of its original value, and usually less than a few percent (see also Bremmer $e t a l .{ }^{20}$ ). Moreover, the sensitivities to eye position usually were lower than the vestibular sensitivities by an order of magnitude. 


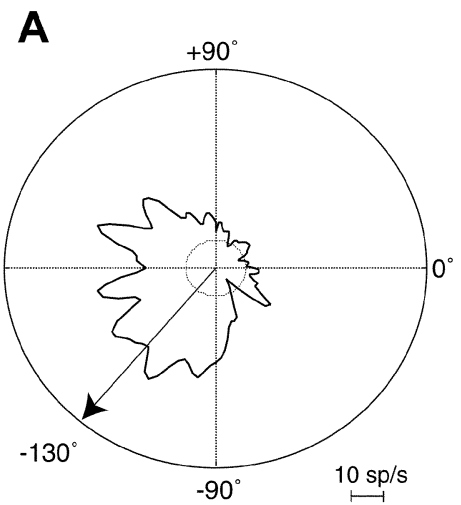

C
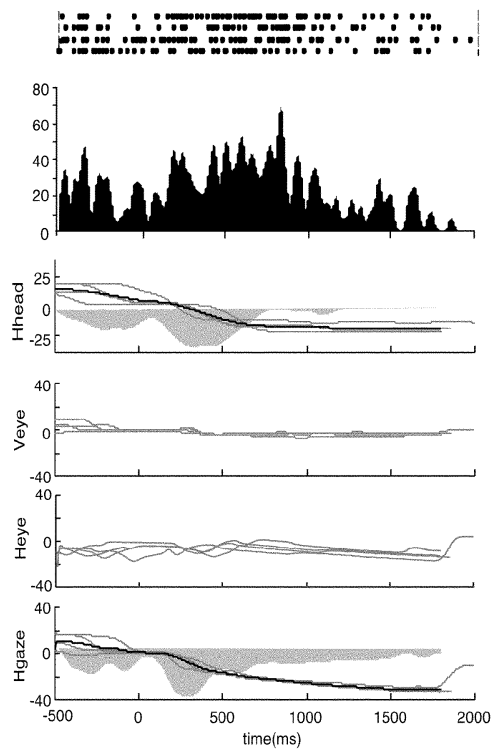

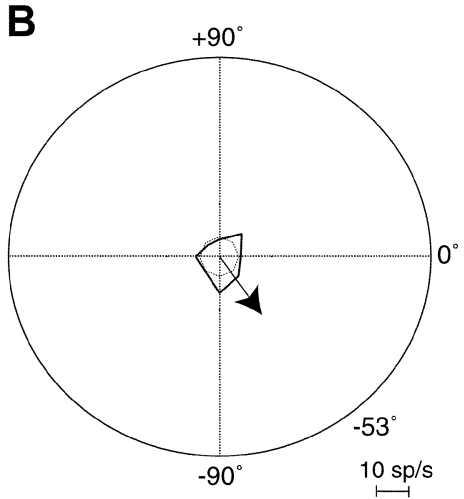

D
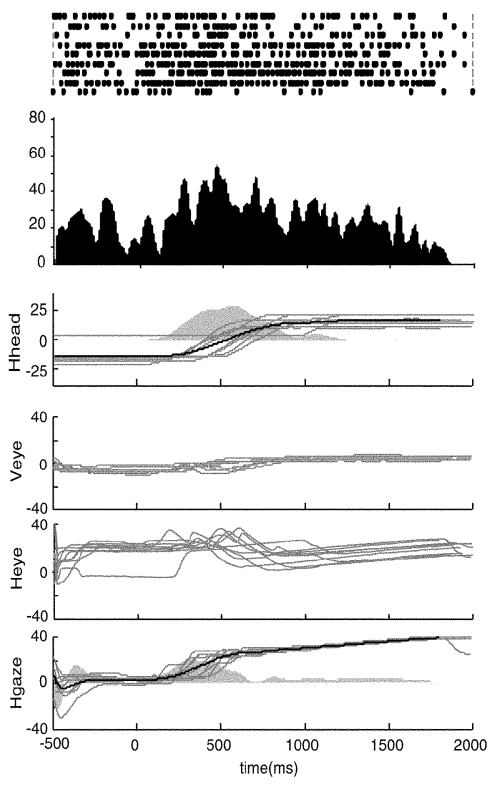

FIGURE 2. Response parameters of a posterior parietal vestibular neuron. (A) Polar plot of visual directional selectivity, indicating a preferred direction into the left hemifield (head-fixed condition). (Solid black line) Neuronal firing rate during stimulation; (dotted circle) resting discharge with fixation spot illuminated. (B) Polar plot of directional tuning during smooth pursuit (head-fixed condition). (Outer solid line) Firing rate during smooth pursuit; (dotted circle) discharge with fixation spot illuminated. (C) Pursuit of a horizontally moving target (head-free condition) to the left. Rows from top to bottom depict neuronal firing rate (shown as a raster plot and as a histogram); horizontal head movement (position: line, velocity: gray shading); vertical eye position; horizontal eye position; horizontal gaze (position: line, velocity: gray shading). (D) Pursuit of a horizontally moving target (headfree condition) to the right. Presentation sequence is as in $\mathbf{C}$. Note that the animal, in this case almost exclusively, uses head movements to pursue the target (in particular in $\mathbf{C}$ ). The neuron's firing rate (vestibular type III) seems to be related principally to head movement. 
Smooth pursuit sensitivities were typically negligible as well (see FIG. 2B). We thus proceeded with our analysis without taking eye-movement effects further into account. Preferred directions of visual stimulus motion were determined using the weighted average method (for details, see Bremmer et al. ${ }^{20,27}$ ). All analyses were performed using either the SAS statistical package or programs in MATLAB and in visual $\mathrm{C}++$.

\section{Anatomical Location of Recorded Cells and Physiological Characterization}

In our experiments, neurons were recorded along microelectrode tracks determined by a grid that allowed reproducible positionings across experimental sessions with maximal precision. While descending in the intraparietal sulcus from the surface, vestibular testing was performed regularly. Besides VIP, we recorded also from MIP, a second intraparietal vestibular zone that was quite distinct from VIP for anatomical location and physiological characteristics. The recording sites have been verified in the fascicularis monkey to be located in the medial bank and in the fundus of the intraparietal sulcus (FIG. 1C). The rhesus monkey is still used in ongoing experiments.

\section{RESULTS}

A total of 106 cells were recorded in the intraparietal sulcus of two left hemispheres of two macaque monkeys in response to various visual, vestibular, and oculomotor paradigms, and active-passive head-movement comparisons.

\section{Posterior Parietal Vestibular Neurons during Pursuit Head Movements}

Posterior parietal vestibular neurons could be shown to be visually directionselective, as reported previously (Bremmer et al.; ${ }^{20,27}$ FIG. 2A). Although all vestibular-responsive posterior parietal neurons had eye-position sensitivity (Bremmer et $\left.a l .{ }^{20}\right)$, smooth pursuit activity was almost negligible at the velocities tested in our experiments (up to $20 \%$ s; FIG. 2B). Under certain circumstances, the animals were tracking the fixation spot almost exclusively via head movements (FIG. 2C, D). In such case, a clear correlation between neuronal firing rate and head movement could be established. Surprisingly, many parietal vestibular neurons showed type III vestibular responses during active head movements (see also FIG. 3C, D).

\section{Comparison between Passive and Active Head Movements}

Response characteristics of parietal vestibular neurons during active and passive head movements were studied by comparing the neuronal firing rate during an active head movement with that of the replay of the same head-movement profile under passive and head-fixed conditions (FIG. 3). The roster of the illustrated neuron examples shows a wide variety of responses, ranging from total extinction of the vestibular signal during active movement (FIG. 3A) to presence of activation only during active movement with absence of any neuronal reaction during passive stimulation (FIG. 3B). Neuronal signals could be diminished in the active movement condition compared with passive stimulation (FIG. 3C), or the vestibular signal could become 
stronger in the active movement condition (FIG. 3D). Most surprisingly, quite frequently we also found a change of directional selectivity for the vestibular on-directions of the recorded neurons (FIG. 3D). The neuron illustrated in FIGURE 3D has a type III response in the active head-movement condition but shows a type I response under passive vestibular stimulation (i.e., excitation via rotation to ipsilateral and vice versa).
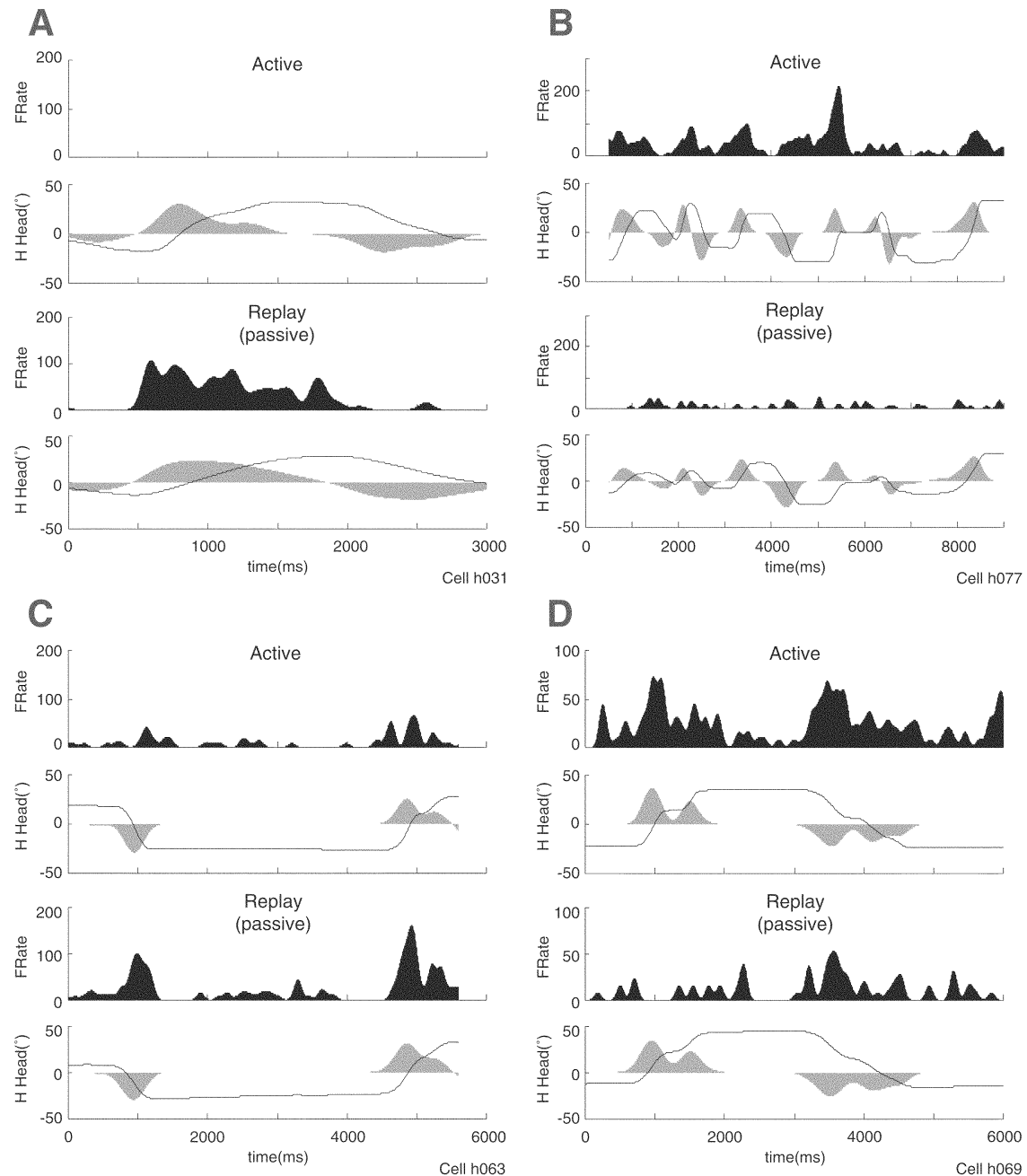

FIGURE 3. Comparison of firing behavior of parietal vestibular neurons during active and passive (replay) head movements. (A) Type II neuron active only during passive rotation. (B) Type II neuron only active during active head movement. (C) Type III neurons whose activity is larger during passive stimulation. (D) Under active movement conditions, this neuron shows type III behavior; under passive stimulation it shows type I behavior. Neuronal activity is greater during active head movements than during passive stimulation. 

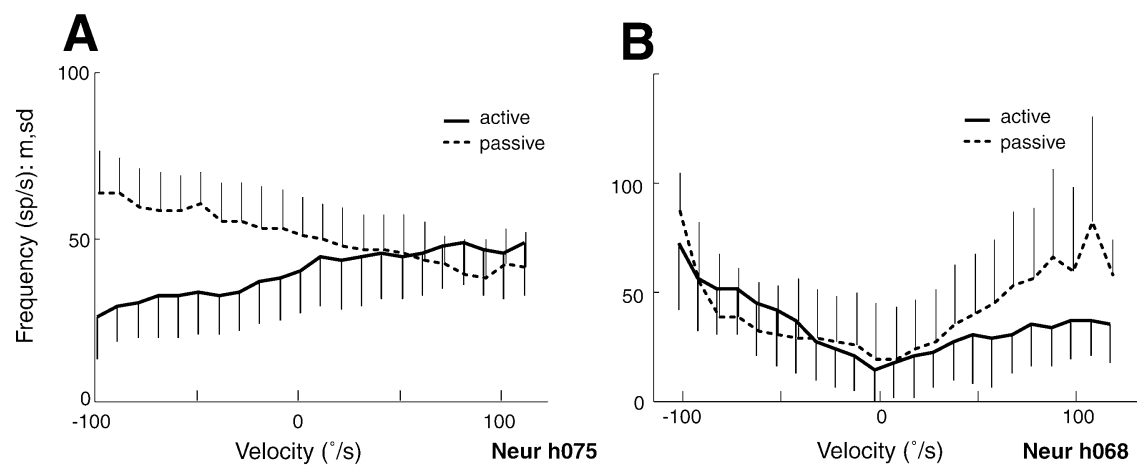

C

Change of vestibular directional selectivity depending on active versus passive mode of movement

\begin{tabular}{c|cc|cc|cc|c} 
& $|\rightarrow| I$ & $|\rightarrow| I \mid$ & $\| \rightarrow \mid$ & $\| \rightarrow|I|$ & $|I \rightarrow| I$ & $\| I \rightarrow \mid$ & \\
\hline monkey 1 & 5 & 2 & 9 & 5 & 3 & 2 & $26(n=68)$ \\
\hline monkey 2 & 4 & 0 & 1 & 1 & 2 & 0 & $7 \quad(n=18)$
\end{tabular}

FIGURE 4. Illustration and quantification of change of on-direction of posterior parietal vestibular neurons during active and passive head movements. (A) Neuronal firing rate in relation to head velocity. The neuron shows type II behavior (increase of firing toward positive velocity values, i.e., rotation to the right) during active head movement and type I behavior (increase of firing toward negative velocity values; i.e., rotation to the left) during passive stimulation. (B) Neuronal firing rate in relation to head velocity. The neuron shows type I behavior (increase of firing toward negative velocity values) during active head movement and type III behavior (increase of firing toward negative and positive velocity values) during passive stimulation. (C) Quantification of neuronal responses. Approximately onethird of the tested neuron population shows a change in vestibular on-direction depending on active versus passive movement mode.

Quantification of change of directional selectivity was provided by plotting firing rates as a function of head velocity of selected neurons during active and passive movement conditions (FIG. 4A, B). In the illustrated examples, one neuron's firing behavior changed from type II in the active condition to type I under passive stimulation (FIG. 4A). In the other case (FIG. 4B), neuronal firing showed type I directional selectivity during active head movements, which then changed to type III behavior under passive stimulation conditions. The full complement of our neuron sample is illustrated in Figure 4C. Clearly, many neurons change directional selectivity depending on active versus passive movement mode, involving all possible combinations of the encountered vestibular response types, that is, types I, II, and III. Of a total of 86 neurons that were tested, 33 (38\%), that is, more than one-third, showed the described change in vestibular on-direction.

Quantification of the strength of neuronal responses under active versus passive movement conditions (FIG. 5) also demonstrated that in most cases, neuronal re- 


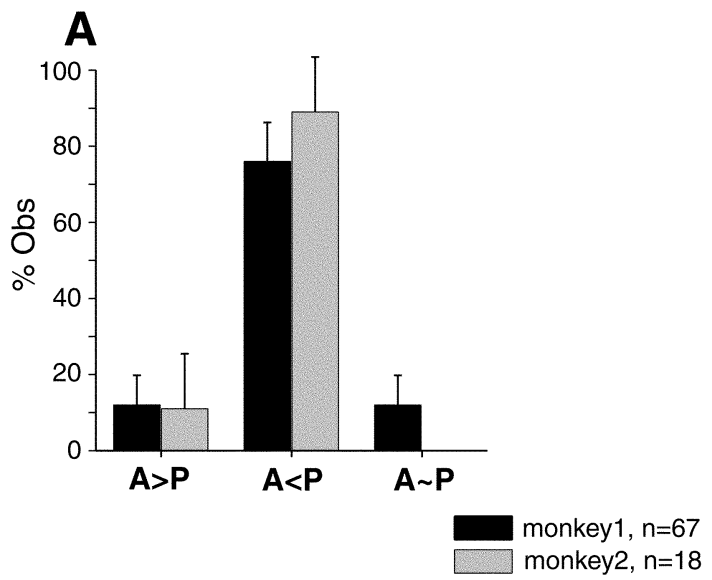

FIGURE 5. Quantification of response strengths of posterior parietal neurons for active or passive movement mode. In most neurons, responses were weaker during active movement than during passive stimulation. However, in a sizable minority, neuronal responses stayed the same or became even stronger during active movement.

sponses were diminished under active movement conditions compared with passive stimulation. In approximately equal proportions, response strengths stayed the same or were even stronger in the active condition.

When determining response delays to vestibular stimulation, neurons clearly had earlier reaction times under active movement condition (FIG. 6). Naturally, under passive stimulation conditions, a given neuron only reacted after the onset of turntable movement. Under active head-movement conditions, neurons could fire as early as $400 \mathrm{~ms}$ before the ensuing head movement, although such cases were rare exceptions. Most neurons fired up to $100 \mathrm{~ms}$ after movement onset. A sizable minority, however, was observed to be activated already up to $100 \mathrm{~ms}$ before the actual head movement.

\section{DISCUSSION}

The variety of vestibular responses in posterior parietal cortex neurons points to a complex processing pattern that is not readily accessible to traditional methods of analysis. Because our present and previous testing (Bremmer et al. ${ }^{20}$ ) had shown little influence of eye-movement signals on the neuronal firing of parietal vestibular neurons, we assume that these neurons are fundamentally different from brainstem vestibular neurons. Their role has to be sought in sensory space representation rather than reflex behavior and motor control contexts. Clearly, each time we perform a head movement, vestibular receptors become activated, and central processing between commands and reflexes takes place. Because vestibular receptors per se cannot distinguish between active and passive movements, ${ }^{29}$ this distinction has to be furnished by central neurons. Second-order vestibular neurons, that is, two synapses 

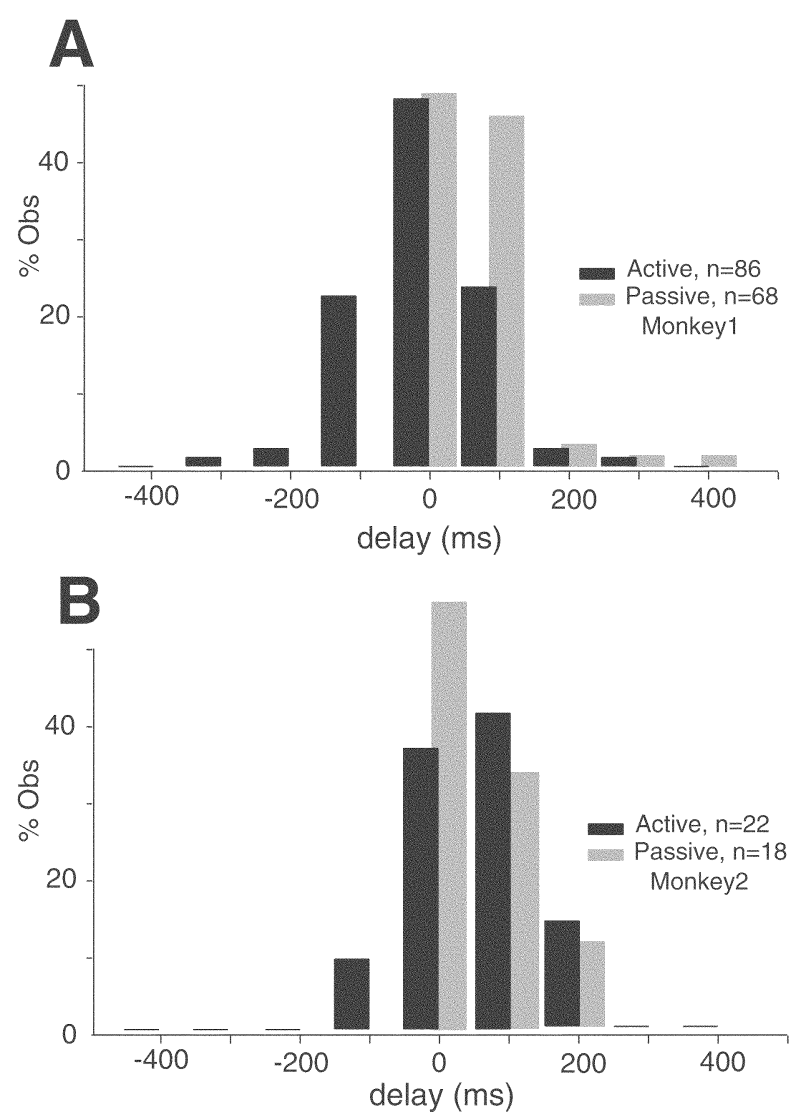

FIGURE 6. Response latencies of neuronal firing rates for onset of head movement. Bin width for analysis was set to $100 \mathrm{~ms}$. Most responses to active head movement and passive stimulation occurred up to $100 \mathrm{~ms}$ after head movement, or stimulus onset. However, neuronal activity could precede self-initiated head movements by as much as $400 \mathrm{~ms}$, although $100 \mathrm{~ms}$ was a value observed more often.

away from the receptor cells already react differentially to active and passive head movements. ${ }^{2-4}$ The question to be answered in this context now is where the actual neuronal processing of this distinction occurs. Posterior parietal cortex neurons may perform this function, or at least play an important role in it. ${ }^{30}$

Although some of our posterior parietal vestibular neurons receive neck input (unpublished observation), the key to understanding active-passive movement distinction processing may be the change in vestibular on-direction of approximately one-third of these neurons (FIG. 4C). A simple combination of neurons with particular directional selectivity depending on active or passive movement mode would allow specific populations of target neurons to become active and thus provide an output signal that would be discriminating between active and passive movement. These signals, in turn, could be used to suppress reflex movements during active 
movement by providing the neural basis for the observed extinction of, for instance, vestibular signals during active head rotation in the vestibular nuclei. ${ }^{2-4}$

A change of on-direction of the vestibular signal depending on the VOR gain was reported in gaze-velocity Purkinje cells, ${ }^{31}$ and such activity has been discussed in context of motor learning. ${ }^{32}$ Our own data point to a much more widespread use of such change in directional selectivity. All in all, we conclude that parietal vestibular neurons may play a much more important role in the processing of self-motion detection signals than has been envisioned up to now, being part of a large network involved the perception of extrapersonal space.

\section{ACKNOWLEDGMENTS}

This work was supported by the European Union (BIO4-CT98-0546), CNRS (UMR 9950/7124), and the ATER program of the Collège de France.

\section{REFERENCES}

1. v. Holst, E. \& H. Mittelstaedt. 1950. Das Reafferenzprinzip. Naturwissenschaften 37: 464-476.

2. McCrea, R.A., G.T. Gdowski, R. Boyle \& T. Belton. 1999. Firing behavior of vestibular neurons during active and passive head movements: vestibulo-spinal and other non-eye-movement related neurons. J. Neurophysiol. 82: 416-428.

3. GDowski, G.T. \& R.A. McCREA. 1999. Integration of vestibular and head movement signals in the vestibular nuclei during whole body rotation. J. Neurophysiol. 81: 436-449.

4. Roy, J.E. \& K.E. CulLEN. 2001. Selective processing of vestibular reafference during self-generated head motion. J. Neurosci. 21: 2131-2142.

5. Matsuo, S., M. Hosogai, H. Matsui \& H. Ikoma. 1995. Posterior canal-activated excitatory vestibuloocular relay neurons participate in the vestibulocortical pathways in cats. Acta Otolaryngol. 520: 97-100.

6. Magnin, M. \& A. Fuchs. 1977. Discharge properties of neurons in the monkey thalamus tested with angular acceleration, eye movement and visual stimuli. Exp. Brain Res. 28: 293-299.

7. BÜtTnER, U., V. Henn \& H.P. Oswald. 1977. Vestibular-related neuronal activity in the thalamus of the alert monkey during sinusoidal rotation in the dark. Exp. Brain Res. 30: 435-444.

8. Grüsser, O.-J., M. PAuse \& U. Schreiter. Vestibular neurons in the parieto-insular cortex of monkeys (Macaca fascicularis): visual and neck receptor responses. J. Physiol. (Lond.) 430: 559-583.

9. Grüsser, O.-J., M. PAUSE \& U. Schreiter. 1990. Localization and responses of neurons in the parieto-insular vestibular cortex of awake monkeys (Macaca fascicularis). J. Physiol. (Lond.) 430: 537-557.

10. SChWARZ, D.W.F. \& J.M. FREDRICKSON. 1971. Rhesus monkey vestibular cortex: a bimodal primary projection field. Science 172: $280-281$.

11. BÜtTNER, U. \& U.W. BuetTNER. 1978. Parietal cortex (2v) neuronal activity in the alert monkey during natural vestibular and optokinetic stimulation. Brain Res. 153: 392 397.

12. ÖDKVIST, L.M., D.W.F. SchwarZ, J.M. FredRICKSON \& R. HASSLER. 1974. Projection of the vestibular nerve to the area $3 \mathrm{a}$ arm field in the squirrel monkey (Saimiri sciureus). Exp. Brain Res. 21: 97-105.

13. Sakata, H., H. Shibutani, Y. Ito, et al. 1994. Functional properties of rotation-sensitive neurons in the posterior parietal association cortex of the monkey. Exp. Brain Res. 101: 183-202. 
14. Colby, C.L. \& J.-R. Duhamel. 1991. Heterogeneity of extrastriate visual areas and multiple parietal areas in the macaque monkey. Neuropsychologia 29: 517-537.

15. Guldin, W.O., S. Akbarian \& O.-J. GrüsSER. 1992. Cortico-cortical connections and cytoarchitectonics of the primate vestibular cortex: a study in squirrel monkeys (Saimiri sciureus). J. Comp. Neurol. 326: 375-401.

16. LEWIS, J.W. \& D.C. VAN ESSEN. 2000. Mapping of architectonic subdivisions in the macaque monkey, with emphasis on parieto-occipital cortex. J. Comp. Neurol. 428: $79-111$.

17. LeWIS, J.W. \& D.C. VAN ESSEN. 2000. Corticocortical connections of visual, sensorimotor, and multimodal processing areas in the parietal lobe of the macaque monkey. J. Comp. Neurol. 428: 112-137.

18. Graf, W., F. Bremmer, S. Ben Hamed \& J.-R. Duhamel. 1996. Visual-vestibular interaction in the ventral intraparietal area (VIP) of macaque monkeys. Soc. Neurosci. Abstr. 22: 666.7.

19. Bremmer, F., J.-R. Duhamel, S. Ben Hamed \& W. Graf. 1997. The representation of movement in near extra-personal space in the macaque ventral intraparietal area (VIP). In Parietal Lobe Contributions to Orientation in 3D Space. P. Thier \& H.O. Karnath, Eds.: 619-630. Springer Verlag. Berlin-Heidelberg-New York.

20. Bremmer, F., F. Klam, J.-R. Duhamel, et al. 2002. Visual-vestibular interactive responses in the macaque ventral intraparietal area (VIP). Eur. J. Neurosci. 16: $1569-1586$.

21. Bremmer, F., W. Graf, S. Ben Hamed \& J.-R. Duhamel. 1999. Eye position encoding in the macaque ventral intraparietal area (VIP). Neuroreport 10: 873-878.

22. Robinson, F.R. \& D.L. Tomko. 1987. Cat vestibular neurons that exhibit different responses to active and passive yaw head rotations. Aviat. Space Environ. Med. 58: A247-249.

23. Colby, C.L., J.-R. Duhamel \& M.E. Goldberg. 1993. The ventral intraparietal area (VIP) of the macaque: anatomical location and visual properties. J. Neurophysiol. 69: $902-914$.

24. SchaAfSMA, S.J. \& J. Duysens. 1996. Neurons in the ventral intraparietal area of awake macaque monkey closely resemble neurons in the dorsal part of the medial superior temporal area in their responses to optic flow patterns. J. Neurophysiol. 76: $4056-4068$.

25. Schatfsma, S.J., J. Duysens \& C.C. Gielen. 1997. Responses in ventral intraparietal area of awake macaque monkey to optic flow patterns corresponding to rotation of planes in depth can be explained by translation and expansion effects. Vis. Neurosci. 14: 633-646.

26. Duhamel, J.-R., C.L. Colby \& M.E. Goldberg. 1998. Ventral intraparietal area of the macaque: congruent visual and somatic response properties. J. Neurophysiol. 79: 126-136.

27. Bremmer, F., S. Ben Hamed, J.-R. Duhamel \& W. Graf. 2002. Optic flow processing in the macaque ventral intraparietal area (VIP). Eur. J. Neurosci. 16: 1554-1568.

28. Duensing, F. \& K.-P. Schaefer. 1958. Die Aktivität einzelner Neurone im Bereich der Vestibulariskerne bei Horizontalbeschleunigungen unter besonderer Berücksichtigung des vestibulären Nystagmus. Arch. Psychiat. Nervenkrankh. 196: 265-290.

29. Cullen, K.E. \& L.B. Minor. 2002. Semicircular canal afferents similarly encode active and passive head-on-body rotations: implications for the role of vestibular efference. J. Neurosci. 22: RC226(1-7).

30. Gabel, S.F., H. Misslich, C.C.A.M. Gielen \& J. Duysens. 2002. Responses of neurons in area VIP to self-induced and external visual motion. Exp. Brain Res. 147: $520-528$.

31. Lisberger, S.G., T.A. Pavelko, H.M. Bronte-Stewart \& L.S. Stone. 1994. Neural basis for motor learning in the vestibuloocular reflex of primates. II. Changes in the responses of horizontal gaze velocity Purkinje cells in the cerebellar flocculus and ventral paraflocculus. J. Neurophysiol. 72: 954-973.

32. Green, A. \& H.L. Galiana. 1996. Exploring sites for short-term VOR modulation using a bilateral model. Ann. N.Y. Acad. Sci. 781: 625-628. 